\title{
Unexplained spectral phenomena in the interstellar medium: an introduction
}

\author{
Sun Kwok ${ }^{1}$ (D)
}

Received: 26 June 2021 / Accepted: 7 July 2021 / Published online: 26 July 2021

(c) The Author(s), under exclusive licence to Springer Nature B.V. 2021

\begin{abstract}
There exists a number of astronomical spectral phenomena that have remained unidentified after decades of extensive observations. The diffuse interstellar bands, the $220 \mathrm{~nm}$ feature, unidentified infrared emission bands, extended red emissions, and 21 and $30 \mu \mathrm{m}$ emission features are seen in a wide variety of astrophysical environments. The strengths of these features suggest that they originate from chemical compounds made of common elements, possibly organic in nature. The quest to understand how such organic materials are synthesized and distributed across the Galaxy represents a major challenge to our understanding of the chemical content of the Universe.
\end{abstract}

Keywords Astrobiology · Astrochemistry · ISM: lines and bands · ISM: molecules · Planetary nebulae: general · Stars: AGB and post-AGB

\section{Historical background}

In 1814, Joseph Fraunhofer observed and tabulated 473 dark lines in the spectrum of the Sun. By comparing the Fraunhofer lines with the bright color lines observed in heated chemical elements, Gustav Kirchhoff and Robert Bunsen identified the solar lines as originating from elements sodium, calcium, magnesium, iron, chromium, nickel, barium, copper, and zinc. This led to the realization that the Sun is made of the same chemical elements as those of the

This article belongs to the Topical Collection: Unexplained Spectral Phenomena in the ISM. Guest Editor: Sun Kwok.

$\triangle$ S. Kwok

skwok@eoas.ubc.ca

1 Department of Earth, Ocean, and Atmospheric Sciences, University of British Columbia, Vancouver, Canada
Earth and marked the demise of the Aristotelian concept of celestial objects being composed of ether.

In 1868, Norman Lockyer discovered a bright yelloworange line in the solar spectrum, which he suggested to be due to a new element and named it "helium". The existence of this new element was confirmed by the discovery of its terrestrial counterpart in 1895. In 1869, a green line was found in the spectrum of the corona of the Sun during the total solar eclipse, and this line was also thought to be a new element and was named "coronium". In 1864, William Huggins found a bright green emission line in the spectrum of the planetary nebula NGC 6543. Since this line did not match any lines from known chemical elements, it was suggested to be due to a new element "nebulium". It was not until the early 20th century that "coronium" was identified as electronic transitions from ionized iron $\left(\mathrm{Fe}^{13+}\right)$ due to the high temperature in the solar corona, and "nebulium" was identified as forbidden lines of ionized oxygen $\left(\mathrm{O}^{++}\right)$arising under low-density nebular conditions. These applications of quantum theory of atoms and laboratory atomic spectroscopy to explain astronomical observations represented the beginning of the modern discipline of astrophysics.

In the early 21 st century, we are facing similar challenges in the form of a number of unexplained spectral phenomena in the interstellar medium. The diffuse interstellar bands (DIBs) were discovered in 1922, when two optical absorption lines of interstellar origin were seen in the spectra of stars (Heger 1922). As of 2021, over 500 DIBs in the ultraviolet, visible, and infrared wavelength regions have been cataloged along the line of sights of over 100 stars.

The $220 \mathrm{~nm}$ ultraviolet feature was discovered in 1965 (Stecher 1965). This feature is seen in the extinction curves of many stars, with characteristically consistent profiles and peak wavelengths. Its wide presence suggests that the carrier is a common constituent of the diffuse interstellar medium. 
The extended red emission (ERE) is a broad $(\Delta \lambda \sim$ $80 \mathrm{~nm}$ ) emission band peaking between 650 and $800 \mathrm{~nm}$, first discovered in the reflection nebula HD44179 (Cohen et al. 1975). ERE has been detected in reflection nebulae, dark nebulae, cirrus clouds, planetary nebulae, H II regions, diffuse interstellar medium, and haloes of galaxies. It is commonly attributed to photoluminescence powered by far UV photons. It is estimated that $\sim 4 \%$ of the energy absorbed by interstellar dust at $\lambda<0.55 \mu \mathrm{m}$ is emitted in the form of the ERE.

A family of unidentified infrared emission (UIE) features at 3.3, 6.2, 7.7, 8.6, and $11.3 \mu \mathrm{m}$ was discovered in the spectrum of the planetary nebula NGC 7027 (Russell et al. 1977). The $3.3 \mu \mathrm{m}$ feature was first identified as the $\mathrm{C}-\mathrm{H}$ stretching mode of aromatic compounds by Knacke (1977). The organic origin of the UIE bands was extensively discussed by Duley and Williams (1981), who assigned the 3.3 and $11.3 \mu \mathrm{m}$ features to graphitic (aromatic) materials.

Also present in astronomical spectra are emission features around $3.4 \mu \mathrm{m}$, which arise from symmetric and antisymmetric $\mathrm{C}-\mathrm{H}$ stretching modes of methyl and methylene groups (Jourdain de Muizon et al. 1990). The bending modes of these groups also manifest themselves at 6.9 and $7.3 \mu \mathrm{m}$. In addition, there are unidentified emission features at $15.8,16.4,17.4,17.8$, and $18.9 \mu \mathrm{m}$. The emission bands themselves are often accompanied by strong, broad emission plateaus features at $6-9,10-15$, and $15-20 \mu \mathrm{m}$. The first two plateau features have been identified as superpositions of inplane and out-of-plane bending modes emitted by a mixture of aliphatic side groups attached to aromatic rings (Kwok et al. 2001). This collection of features in the UIE family has been observed in planetary nebulae, reflection nebulae, novae, $\mathrm{H}$ II regions, and galaxies.

The unidentified infrared emission feature around $30 \mu \mathrm{m}$ was discovered from Kuiper Airborne Observatory observations (Forrest et al. 1981). It was first seen in carbon-rich asymptotic giant branch stars, planetary nebulae, and protoplanetary nebulae. Among planetary nebulae in the Magellanic Clouds, about half of them possess the $30 \mu \mathrm{m}$ feature (Bernard-Salas et al. 2009).

The $21 \mu \mathrm{m}$ emission feature was first discovered in Infrared Astronomical Satellite Low Resolution Spectroscopic survey (Kwok et al. 1989). The feature peaks around $20.1 \mu \mathrm{m}$ and shows a broad $(\sim 2 \mu \mathrm{m})$ and smooth profile. The $21 \mu \mathrm{m}$ feature is almost always accompanied by the $30 \mu \mathrm{m}$ feature. The $21 \mu \mathrm{m}$ feature is primarily observed in carbon-rich post-asymptotic-giant-branch stars. From the spectral energy distribution of $21 \mu \mathrm{m}$ sources, it is found that the 21 and $30 \mu \mathrm{m}$ features can carry respectively up to 8 and $20 \%$ of the total energy output of the objects (Hrivnak et al. 2000).

\section{Distribution in the Universe}

Although these unexplained spectral phenomena were first discovered in the spectra of stars, they are not isolated phenomena as they are observed in a wide range of celestial objects throughout the Universe. DIBs have been detected in external galaxies with redshifts up to 0.5 (Sarre 2006). The $220 \mathrm{~nm}$ feature has been detected in interplanetary dust particles in the Solar System (Bradley et al. 2005) as well as in distant galaxies with redshift $>2$ (Elíasdóttir et al. 2009). A survey of 150 galaxies by the AKARI satellite found that $\sim 0.1 \%$ of the total energy of the parent galaxies is emitted through the $3.3 \mu \mathrm{m}$ UIE band (Imanishi et al. 2010). In some active galaxies, up to $20 \%$ of the total luminosity of the galaxy is emitted in the UIE bands (Smith et al. 2007). The $3.4 \mu \mathrm{m}$ aliphatic feature has been detected in absorption in ultraluminous infrared galaxies (Mason et al. 2004; Risaliti et al. 2006). The detection of UIE bands in highredshift galaxies (Teplitz et al. 2007) and quasars (Lutz et al. 2007) implies that organic compounds were widely present as early as 10 billion years ago. This suggests that abiological synthesis of complex organics was active through most of the history of the Universe.

\section{Chemical nature of the carriers}

Because of the strengths and ubiquitous nature of the features, the carrier must be made of common, abundant elements, with the element carbon probably playing a major role. While the DIBs are commonly believed to be due to electronic transitions of gas-phase carbon-based molecules, the carrier of the $220 \mathrm{~nm}$ feature is more likely to be a carbonaceous solid such as amorphous carbon (Mennella et al. 1998), carbon onions (Iglesias-Groth 2004), hydrogenated fullerenes (Cataldo and Iglesias-Groth 2009), or polycrystalline graphite (Papoular and Papoular 2009). Among the hundreds of DIBs, only two ( 963.2 and $957.7 \mathrm{~nm}$ ) have been positively identified as originating from ionized fullerene $\left(\mathrm{C}_{60}^{+}\right.$, Foing and Ehrenfreund 1994; Campbell et al. 2015). Two weaker lines of $\mathrm{C}_{60}^{+}$at 942.8 and $936.6 \mathrm{~nm}$ have also been suggested to have counterparts in DIBs (Walker et al. 2015).

A variety of chemical structures have been suggested as the carriers of the UIE bands. These include polycyclic aromatic hydrocarbon (PAH) molecules (Léger and Puget 1984; Allamandola et al. 1989), small carbonaceous molecules (Bernstein and Lynch 2009), hydrogenated amorphous carbon (HAC), soot and carbon nanoparticles (Hu and Duley 2008), quenched carbonaceous composite particles (QCC, Sakata et al. 1987), coal and kerogen (Papoular et al. 1989; Papoular 2001), petroleum fractions (Cataldo et al. 2002), and mixed aromatic/aliphatic organic nanoparticles (MAON, Kwok and Zhang 2013). 
Proposed carriers for the $21 \mu \mathrm{m}$ feature include hydrogenated fullerenes (Webster 1995), titanium carbide (von Helden et al. 2000), silicon carbide (Speck and Hofmeister 2004), and thiourea groups attached to aromatic/aliphatic structures (Papoular 2011). Since ERE is the result of photoluminescience, the carrier is likely a semiconductor with a nonzero band gap. Other proposed carbon-based carriers include QCC (Sakata et al. 1992), $\mathrm{C}_{60}$ (Webster 1993), and nanodiamonds (Chang et al. 2006).

\section{Future outlook}

In this topical collection, the recent developments in our understanding of the ERE and the 21/30 $\mu \mathrm{m}$ features are reviewed by Witt and Lai (2020), and Volk et al. (2020) respectively. The possible ERE-DIB connection is also discussed by Witt and Lai (2020). The scenario of coal and petroleum related compounds as carriers of UIE is discussed by Cataldo et al. (2020). The possibilities of hydrogenated fullerenes as carriers of DIB, ERE, and $220 \mathrm{~nm}$ features are reviewed by Zhang et al. (2020).

Is it possible that these unexplained spectral phenomena be related to each other? The identification of two DIBs with $\mathrm{C}_{60}^{+}$suggests the possibility that the DIB carrier molecules may be breakdown products of large, complex organic compounds, such as MAONs or other amorphous hydrocarbons. The presence of the 8 and $12 \mu \mathrm{m}$ UIE plateau features in fullerene sources also suggests that fullerenes and UIE carriers may share common precursors.

While we do not know when and where the carriers of DIBs and $220 \mathrm{~nm}$ features are synthesized, the observations of UIE bands, ERE, and 21/30 $\mu \mathrm{m}$ features in the circumstellar environment dictate that the chemical synthesis time scale is constrained by the evolutionary and dynamical time scale of the circumstellar envelopes. For example, UIE bands are observed to emerge over time scales of $\sim 10^{3}$ years in the proto-planetary nebulae phase (Kwok et al. 1999), and over time scales of weeks in novae (Helton et al. 2011). This suggests that the synthesis of the UIE carriers is extremely efficient. How such synthesis can occur so rapidly under low-density conditions is not understood by our current chemical models.

Could the carriers of these unexplained spectral phenomena be new chemical compounds unobserved on Earth, as in the case of the discovery of helium in the Sun, or could they be the result of unusual physical environment as in the case of "coronium" and "nebulium"? Further development in laboratory spectroscopy will hold the key to the identification of the carriers.

Snow (2014) has suggested that DIBs alone represent the largest reservoir of organic material in the Galaxy, so no matter what the exact nature of the carriers is, they must be an important constituent of the Cosmos. The resolution of these spectral mysteries may bring about a new view of our understanding of the chemical content of the Universe.

Acknowledgements This work is supported by a grant from the Natural and Engineering Research Council of Canada.

Publisher's Note Springer Nature remains neutral with regard to jurisdictional claims in published maps and institutional affiliations.

\section{References}

Allamandola, L.J., Tielens, A.G.G.M., Barker, J.R.: Astrophys. J. Suppl. 71, 733 (1989)

Bernard-Salas, J., Peeters, E., Sloan, G.C., et al.: Astrophys. J. 699, 1541 (2009)

Bernstein, L.S., Lynch, D.K.: Astrophys. J. 704, 226 (2009)

Bradley, J., Dai, Z.R., Erni, R., Browning, N., Graham, G., Weber, P., Smith, J., Hutcheon, I., Ishii, H., Bajt, S., Floss, C., Stadermann, F., Sandford, S.: Science 307, 244 (2005)

Campbell, E.K., Holz, M., Gerlich, D., Maier, J.P.: Nature 523, 322 (2015)

Cataldo, F., Iglesias-Groth, S.: Mon. Not. R. Astron. Soc. 400, 291 (2009)

Cataldo, F., Keheyan, Y., Heymann, D.: Int. J. Astrobiol. 1, 79 (2002)

Cataldo, F., García-Hernández, D.A., Manchado, A.: Astrophys. Space Sci. 365, 81 (2020)

Chang, H.-C., Chen, K., Kwok, S.: Astrophys. J. Lett. 639, L63 (2006)

Cohen, M., Anderson, C.M., Cowley, A., et al.: Astrophys. J. 196, 179 (1975)

Duley, W.W., Williams, D.A.: Mon. Not. R. Astron. Soc. 196, 269 (1981)

Elíasdóttir, Á., et al.: Astrophys. J. 697, 1725 (2009)

Foing, B.H., Ehrenfreund, P.: Nature 369, 296 (1994)

Forrest, W.J., Houck, J.R., McCarthy, J.F.: Astrophys. J. 248, 195 (1981)

Heger, M.L.: Lick Obs. Bull. 10, 141 (1922)

Helton, L.A., Evans, A., Woodward, C.E., Gehrz, R.D.: EAS Publ. Ser. 46, 407 (2011)

Hrivnak, B.J., Volk, K., Kwok, S.: Astrophys. J. 535, 275 (2000)

Hu, A., Duley, W.W.: Astrophys. J. Lett. 677, L153 (2008)

Iglesias-Groth, S.: Astrophys. J. Lett. 608, L37 (2004)

Imanishi, M., Nakagawa, T., Shirahata, M., Ohyama, Y., Onaka, T.: Astrophys. J. 721, 1233 (2010)

Jourdain de Muizon, M., D'Hendecourt, L.B., Geballe, T.R.: Astron. Astrophys. 235, 367 (1990)

Knacke, R.F.: Nature 269, 132 (1977)

Kwok, S., Zhang, Y.: Astrophys. J. 771, 5 (2013)

Kwok, S., Volk, K.M., Hrivnak, B.J.: Astrophys. J. Lett. 345, L51 (1989)

Kwok, S., Volk, K., Hrivnak, B.J.: Astron. Astrophys. 350, L35 (1999)

Kwok, S., Volk, K., Bernath, P.: Astrophys. J. Lett. 554, L87 (2001)

Léger, A., Puget, J.L.: Astron. Astrophys. 137, L5 (1984)

Lutz, D., et al.: Astrophys. J. Lett. 661, L25 (2007)

Mason, R.E., Wright, G., Pendleton, Y., Adamson, A.: Astrophys. J. 613, 770 (2004)

Mennella, V., Colangeli, L., Bussoletti, E., Palumbo, P., Rotundi, A.: Astrophys. J. Lett. 507, L177 (1998)

Papoular, R.: Astron. Astrophys. 378, 597 (2001)

Papoular, R.: Mon. Not. R. Astron. Soc. 415, 494 (2011)

Papoular, R.J., Papoular, R.: Mon. Not. R. Astron. Soc. 394, 2175 (2009)

Papoular, R., Conrad, J., Giuliano, M., Kister, J., Mille, G.: Astron. Astrophys. 217, 204 (1989) 
Risaliti, G., Maiolino, R., Marconi, A., et al.: Mon. Not. R. Astron. Soc. 365, 303 (2006)

Russell, R.W., Soifer, B.T., Willner, S.P.: Astrophys. J. Lett. 217, L149 (1977)

Sakata, A., Wada, S., Onaka, T., Tokunaga, A.T.: Astrophys. J. Lett. 320, L63 (1987)

Sakata, A., et al.: Astrophys. J. 393, L83 (1992)

Sarre, P.J.: J. Mol. Spectrosc. 238(1), 1 (2006)

Smith, J.D.T., et al.: Astrophys. J. 656, 770 (2007)

Snow, T.P.: In: Cami, J., Cox, N.L.J. (eds.) IAU Symposium 297:

The Interstellar Diffuse Bands, p. 3. Cambridge University Press, Cambridge (2014)

Speck, A.K., Hofmeister, A.M.: Astrophys. J. 600, 986 (2004)
Stecher, T.P.: Astrophys. J. 142, 1683 (1965)

Teplitz, H.I., et al.: Astrophys. J. 659, 941 (2007)

Volk, K., Sloan, G.C., Kraemer, K.E.: Astrophys. Space Sci. 365, 88 (2020)

von Helden, G., et al.: Science 288, 313 (2000)

Walker, G.A.H., Bohlender, D.A., Maier, J.P., Campbell, E.K.: Astrophys. J. Lett. 812, L8 (2015)

Webster, A.: Mon. Not. R. Astron. Soc. 264, L1 (1993)

Webster, A.: Mon. Not. R. Astron. Soc. 277, 1555 (1995)

Witt, A.N., Lai, T.S.-Y.: Astrophys. Space Sci. 365, 58 (2020)

Zhang, Y., Sadjadi, S., Hsia, C.-H.: Astrophys. Space Sci. 365, 67 (2020) 